\title{
Mixed Conventional and Desmoplastic Infantile Ganglioglioma: an Autopsied Case with 6-Year Follow-Up
}

Takashi Komori, M.D., Ph.D, Bernd W. Scheithauer, M.D., Joseph E. Parisi, M.D., Jan Watterson, B.A., John R. Priest, M.D.

Department of Pathology and Laboratory Medicine, Mayo Clinic, Rochester, Minnesota (TK, BWS, JEP); Department of Clinical Neuropathology, Tokyo Metropolitan Institute for Neuroscience, Fuchu, Tokyo

(TK); and Children's Hospital of St. Paul, St. Paul, Minnesota (JW, JRP)

We describe a case of desmoplastic infantile ganglioglioma (DIG) arising in the ventral diencephalon of a 3-1/2-month-old boy. On biopsy, the tumor featured a desmoplastic, S-100 protein and GFAP immunoreactive stromal element, as well as a variable spectrum of ganglion cells. Electron microscopy demonstrated astrocytes, and morphologically fibroblasts, as well as neurons containing $120-\mathrm{nm}$ dense core granules. In addition, tubular structures composed of tightly apposed cells with features of astrocytes and of Schwann-like cells were also noted. Devoid of fibroblasts, the tubular structures were surrounded by a single basal lamina. At autopsy 6 years later, the multinodular, cystic mass had replaced the diencephalon, extended into both temporal lobes as well as the optic nerves, and showed marked leptomeningeal involvement. Microscopically, superficial portions of the tumor consisted of typical DIG, whereas deep, nondesmoplastic portions exhibited pattern variation ranging from pilocytic astrocytoma to ganglioglioma and gangliocytoma. There was also a minor element of small, 'primitive-appearing' neuroepithelial cells. Dysplastic ganglion cells variously reactive for neurofilament protein and synaptophysin were present throughout the tumor. Our study not only confirms DIG as a variant of ganglioglioma, one capable of slow growth, infiltration, and fatal progression but suggests that its differen-

Copyright (C) 2001 by The United States and Canadian Academy of Pathology, Inc.

VOL. 14, NO. 7, P. 720, 2001 Printed in the U.S.A.

Date of acceptance: February 27, 2001.

Presented in part at the 68th Annual Meeting of the American Association of Neuropathologists, St. Louis, Missouri, on June 18, 1992.

Address reprint requests to: Bernd W. Scheithauer, M.D., Department of Pathology and Laboratory Medicine, Mayo Clinic, 200 First Street SW, Rochester, MN 55905; e-mail: scheithauer.bernd@mayo.edu; fax: 507-284-1599. tiating potential includes elements of both the central and peripheral nervous systems. If so, their derivation may be from multipotential cells of the neural plate.

KEY WORDS: Desmoplastic infantile ganglioglioma (DIG), Divergent differentiation, Ganglioglioma, Schwann cell.

Mod Pathol 2001;14(7):720-726

Desmoplastic infantile ganglioglioma (DIG) is a rare pediatric brain tumor characterized by divergent glioneuronal differentiation and intense desmoplasia $(1,2)$. Superficial in location and affecting mainly the frontotemporal region, it presents as a large, cystic, often dura-attached mass in patients younger than 2 years of age. Pericellular basal lamina formation is a characteristic of its spindled glial cells. Although a generally well accepted entity, the origin and cellular makeup of DIG are incompletely understood. In the most recent edition of the World Health Organization (WHO) classification of tumors of the nervous system, DIG and desmoplastic infantile astrocytoma (DIA) (3) are listed together as desmoplastic infantile astrocytoma/ganglioglioma (DIA/DIG; 4). The occurrence of Schwann cell differentiation, a possible feature of the present case, had also been suggested in an earlier case report of DIG (5). Although the prognosis of DIG is generally excellent after resection alone (4), evaluations of autopsied cases with long-term survivals have not been described. Herein, we compare the biopsy and autopsy findings of a fatal case of DIG after a six-year interval of slow but progressive growth. In addition to classic DIG, the tumor exhibited a conventional ganglioglioma component as well as unusual features suggesting Schwann cell differentiation. 


\section{CASE REPORT}

A 2-month-old male exhibited rapid head enlargement, irritability, and occasional emesis. Normal in weight, he was the product of a full-term pregnancy and an uneventful delivery. The family history was noncontributory, and there was no evidence of neurofibromatosis. At age $31 / 2$ months, he was alert but had a tense fontanel and showed poor head control. Range of motion was full, and cranial nerve function was normal. Reflexes were brisk and increased in the upper extremities. A poor grasp reflex was also noted. A computed tomography (CT) scan of the head (Fig. 1A) revealed a cystic and heterogeneously enhancing, $7-\mathrm{cm}$ tumor involving the left hypothalamic, third ventricular, and bilateral thalamic regions. In addition, it involved the inferomedial, frontal, and temporal lobes on the left, just reaching the lateral aspect of the temporal lobe. Solid, contrast-enhancing portions of the tumor involved mainly the ventral, diencephalon and the medial aspect of middle temporal lobe. A fourvessel angiogram showed the tumor to be avascular. At left temporal craniotomy, grape-like, fluidfilled cysts were seen to occupy the tip of the temporal fossa. There was no apparent involvement of the dura. A biopsy was taken of fibrousappearing tissue deep within the temporal fossa and extrinsic to brain parenchyma. The tumor's extent precluded a complete resection. Right and left lateral ventricular as well as subduralabdominal shunts were placed. Despite a 3-month cycle of vincristine and actinomycin D chemotherapy, no neurologic or radiographic improvement was noted. Infantile spasms developed at age 6 months, an electroencephalogram revealing simultaneous hypsarrhythmia. Between 5 and 9 months of age, the patient's course was marked by the recurring episodes of massive ascites; cytologic preparations were free of neoplastic cells. Shunts

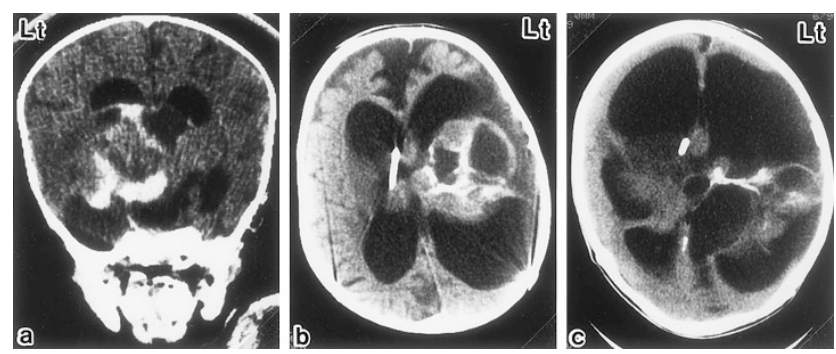

FIGURE 1. Serial CT scans. A, Enhanced scan at age $31 / 2$ month showing the heterogeneous tumor to occupy the thalamic area and to bulge into the left lateral ventricle as well as the interpeduncular fossa. Note intense enhancement in ventral hypothalamic portions of the tumor (coronal view). B, Unenhanced scan at age 4 years showing enlargement of the cystic mass. A ventricular shunt is in place. $\mathbf{C}$, Unenhanced scan at age 6 years ( 4 months before death) showing the mass to occupy the right diencephalon. Compared with the prior scans, cysts and hydrocephalus have progressed, but solid portions of the tumor have not. were occasionally revised. By 18 months, the patient had developed a palmar grasp, responded to sounds, occasionally vocalized, appeared to reach out to presented stimuli, and could mouth as well as swallow small quantities of soft food. At age 25 months, a CT scan showed tumor enlargement as well as its extension into the right diencephalon (Fig. 1B). Over the ensuing years, the child showed neither intellectual nor psychosocial development. Eventually dependent upon gastrostomy feeding, his neurologic status slowly deteriorated. Sequential CT scans showed slow growth of solid components of the tumor, progression of the cysts, increasing ventricular size, and atrophy of the cortical mantle (Fig. 1C). At age 6 years, marked temperature instability and shallow respiration developed over a period of several months. Death was due to respiratory insufficiency. An autopsy was performed 5 hours thereafter.

\section{MATERIALS AND METHODS}

All specimens were fixed in $10 \%$ neutral buffered formalin and were routinely processed for light microscopy. Five-micrometer sections were stained by the hematoxylin and eosin (H\&E), reticulin, and Masson's trichrome methods and were immunohistochemically studied using the streptavidinbiotin peroxidase complex (ABC) method. Antisera applied were directed against glial fibrillary acid protein (dilution 1:300; DAKO, Carpinteria, CA), S-100 protein (1:800; HSC, Toronto, Canada), vimentin (1:20; DAKO), synaptophysin (SYP) (1:40; ICN, Costa Mesa, CA and 1:100; DAKO), MAP-2 (1:400; Boehringer, Mannheim, Germany), neurofilament protein (NFP; 1:75; Dako), collagen IV (1: 100, DAKO), chromogranin A (1:100, DAKO), and the Ki-67 antigen MIB-1 (1:200; AMAC Corporation, West Brook, ME). The Ki-67 labeling index (LI), expressed as percentage positive nuclei selected in five high-power fields demonstrating maximal labeling, was quantitated on a CAS 200 image analyzer (Becton-Dickinson, San Jose, CA). For electron microscopy, the biopsy specimen was in fixed in glutaraldehyde, postfixed in osmium tetroxide, stained en bloc with uranyl acetate, and embedded in Spurr resin. Thin sections stained with lead citrate were examined on a JEOL 1220 electron microscope.

\section{RESULTS}

\section{Biopsy Specimen}

\section{Light Microscopic Findings}

Histologically, the multiple tissue fragments showed similar histologic features, consisting mainly of spindle and occasional plump tumor cells 
disposed in fascicular and storiform patterns within a variably dense, Masson's trichrome positive collagenous stroma (Fig. 2A). Other, more lightly stained cells were heterogeneous in appearance and ranged from astrocytic to ganglion cell-like (Fig. 2A). The former were elongate or stellate, possessed eosinophilic cytoplasm, and contained heterochromatic nuclei. In contrast, the ganglion-like cells featured plump cell bodies vesicular euchromatic nuclei and single, small nuclei (Fig. 2A). No mature ganglion cells were identified. Multinucleate cells were rare. These subtypes of tumor cells were also evident in toluidine blue-stained semithin sections (Fig. 2B). Overall, cellularity and pleomorphism were moderate. Mitoses were very rare.

Regionally, a distinctive feature was apparent, the formation of compact tubular or insular structures surrounded by an amorphous eosinophilic sheath and fibroblasts (Fig. 2B). Whereas generally the tumor stroma consisted of Masson's trichrome positive collagen and reticulin, tubular structures were free of connective tissue (Fig. 2C). Immunoreactivity for GFAP and S-100 protein was mainly noted within the tubular structures referred to above but was also seen within the cytoplasm and processes of single cells embedded in the stroma (Fig. 2D). No staining was apparent in obvious fibroblasts. Collagen IV reactivity was mainly seen around the ves-

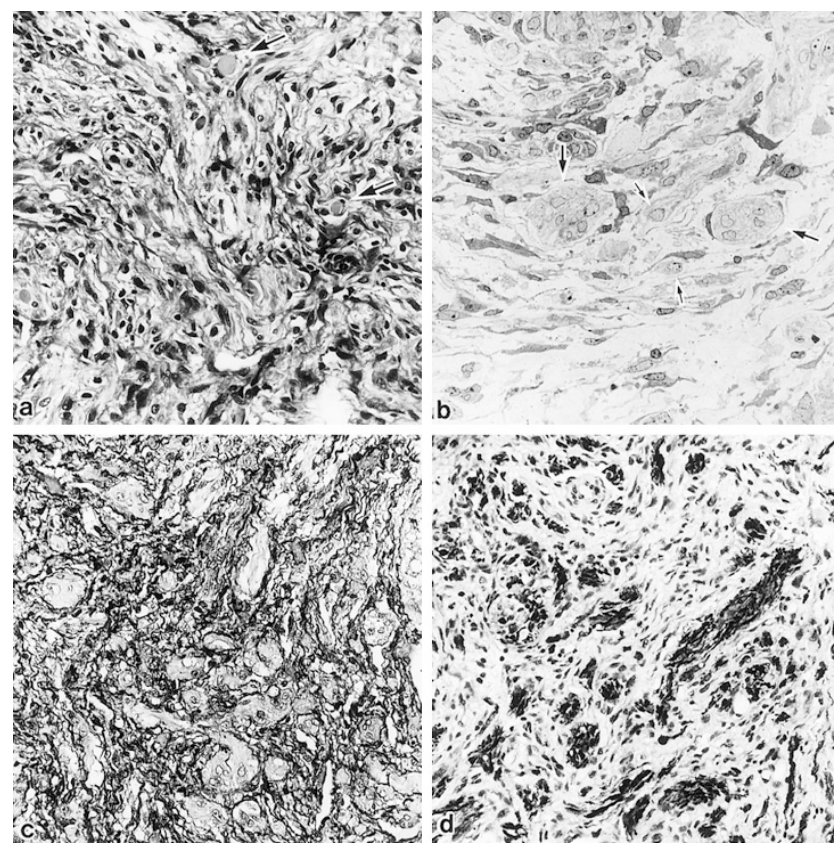

FIGURE 2. Biopsy specimen at $31 / 2$ months of age. A, A fascicular and somewhat storiform pattern of growth typifies the tumor. Occasional ganglion-like cells are evident (arrows) (H\&E). B, A 1- $\mu \mathrm{m}$ toluidine blue-stained preparation demonstrates tubular structures (large arrows) surrounded by dark, bipolar fibroblasts with elongate processes. Smaller arrows indicate probable neuronal or "ganglion-like" cells within the stroma. C, Reticulin staining shows intense desmoplasia. Note that tubular and insular structures lack staining. D, GFAP immunoreactivity is present within tubular structures and in a fraction of the spindle cells enmeshed in collagenous stroma. sels. Vimentin immunoreactivity was found in all but ganglion-like cells, some of which lay isolated in stroma and were positive for SYP and/or MAP-2. Neither NFP nor chromogranin A immunoreactivity was seen. Also lacking was chromogranin-A mRNA. The Ki-67 LI was a very low $0.36 \%$.

\section{Ultrastructural Findings}

Because of sampling, the specimen consisted primarily of the above-noted tubular or insular structures and of type I collagen-rich stroma containing fibroblasts. Bipolar and electron-dense fibroblasts featured often crenated nuclei, tapering processes, and abundant dilated rough endoplasmic reticulum (RER) but were devoid of basal lamina and pinocytic vesicles (Fig. 3A). Also embedded in the stroma were occasional neuronal cells with oval, euchromatic nuclei; prominent nucleoli; and abundant cytoplasm containing profiles of RER, welldeveloped Golgi, mitochondria, bundles of microtubules, small numbers of intermediate filaments, and sparse, $120 \mathrm{~nm}$ dense core granules (DCG) (Fig. 3B). Although aggregates of subplasmalemmal clear vesicles were occasionally seen in cell bodies or processes, no definite vesicle-associated synapses were identified. Also present were numerous axonal spheroids, containing randomly arrayed intermediate filaments, mitochondria, smooth ER, and scattered DCG. Astrocytic cells were rare outside the tubes and islands, but occasionally possessed an incomplete basal lamina. No pinocytotic vesicles were found on the surfaces of the cells within the stroma. The above-noted tubular and insular structures were well represented and consisted of tightly apposed tumor cells and processes (Fig. 4A) but neither fibroblasts nor collagen fibers. Although in-

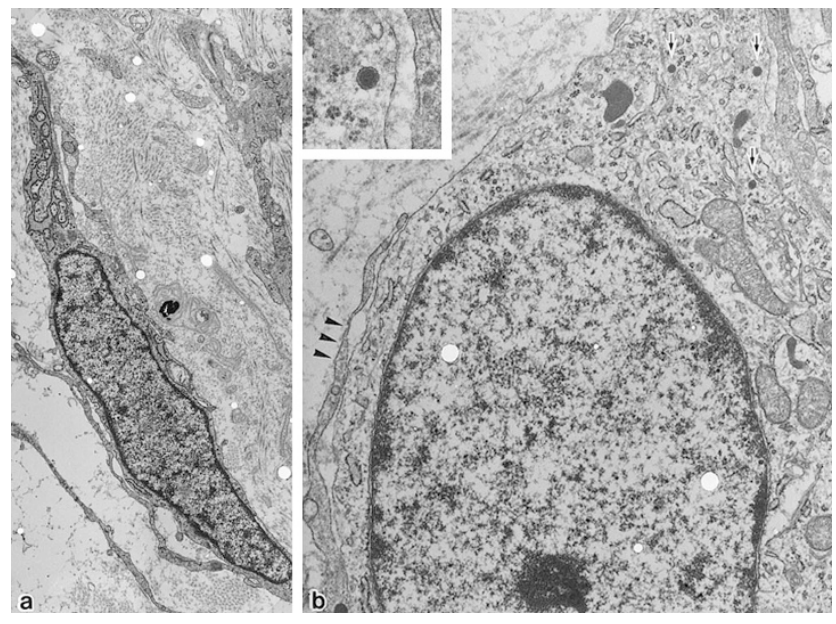

FIGURE 3. A, A stromal fibroblast with its bipolar configuration and characteristic dilated rough endoplasmic reticulum $(7,300 \times)$. B, A ganglion cell containing microtubules, mitochondria, Golgi, and occasional dense core granules (DCG, arrow); 17,300×). Note lack of a basal lamina and its intimate association with a fibroblast process (arrowheads). The inset shows a typical 130-nm DCG $(36,000 \times)$. 
dividual intratubular cells lacked a basal lamina, the tubes were uniformly ensheathed by a single layer of continuous basal lamina. Fibroblasts surrounding the tubes and islands never showed direct attachment to their surfaces. It was difficult to determine the nature of the individual character cells within the tubes. Some resembled astrocytes as evidenced by scant organelles, densely packed intermediate filaments, and small punctate adhesions. On the other hand, small numbers showed features suggesting Schwann cell differentiation, being engaged in the formation of mesaxon-like structures. Cytoplasm indentations and thin, branched cytoplasmic processes enclosed or ensheathing axons containing parallel arrays of microtubules (Fig. 4AB). These Schwann-like cells possessed relatively abundant cytoplasm containing intermediate filaments and a few microtubules. In addition, occasional primitive-appearing cells featured surface pinocytic vesicles (Fig. 4C).
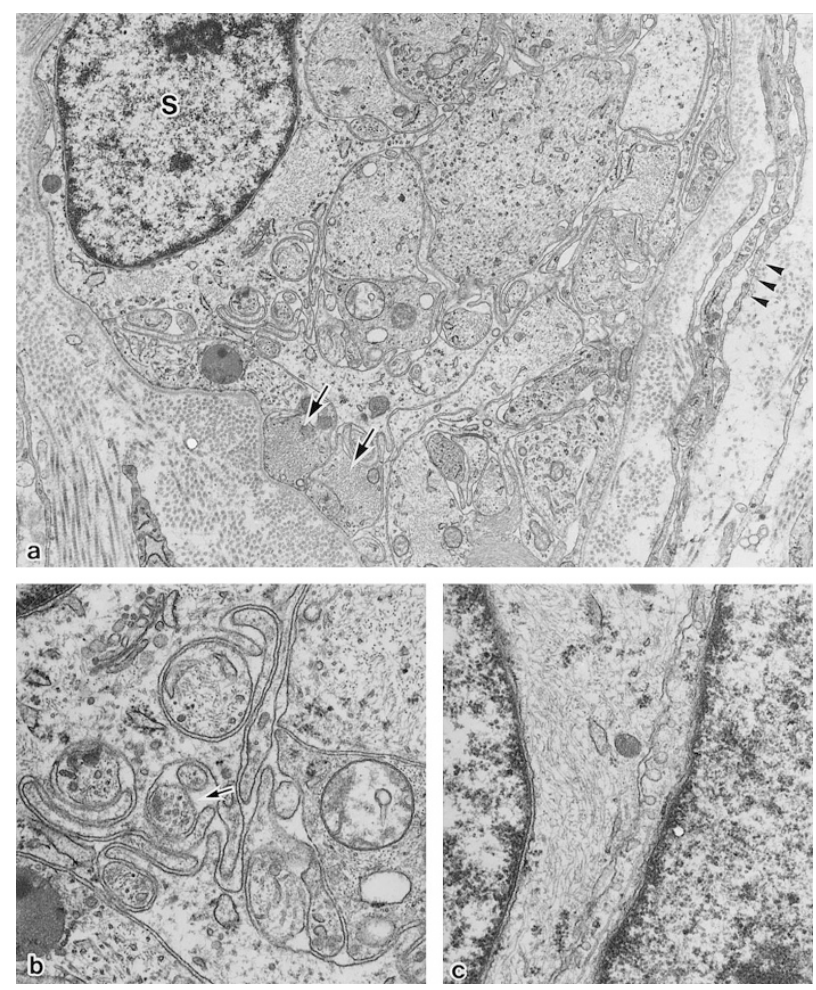

FIGURE 4. A, A cross-sectional view of a tubular structure surrounded by a continuous basal lamina and containing multiple astrocytic and neuronal processes. The arrows indicate astrocytic processes. Note lack of fibroblasts and of collagen fibers within the tube. The cell with a heterochromatic nuclei (S) is covered by basal lamina and shows the formation of mesoaxon-like structures. Note banded interstitial collagen fibrils (lower left) and a close association with fibroblastic processes (arrowheads; 12,500×). B, A higher power view of the mesoaxon-like structures. Note encirclement of microtubule-containing axons (arrow, 31,500×). C, Two adjacent cells within a tube. That on the right shows pinocytic vesicles along its surface, whereas the cell on the left features abundant intermediate filaments but lacks vesicles $(30,000 \times)$.

\section{Autopsy Specimen}

Grossly, the cerebral convexities were densely adherent to overlying dura. The cortical mantle was quite thin, gyri were flattened, and the ventricles were markedly dilated. The tumor protruded ventrally and, forming multiple nodules, entirely obliterated the interpenducular cistern. Both optic nerves, particularly the left $(1.0 \mathrm{~cm}$ diameter), were massively infiltrated. The upper brain stem was compressed, and the cerebellar vermis was displaced to the left. Coronal slices showed extensive replacement of the diencephalon and basal ganglia, more so on the left, by a poorly demarcated, cystic, multinodular, and partly translucent mass (Fig. 5). It was largely white, inhomogeneous in texture, and firm. No distinct cleavage plane was evident between the lesion and surrounding brain tissue.

Microscopically, leptomeningeal involvement was extensive on the ventrolateral aspect of the brain, a location where desmoplasia was prominent and the histologic features were those of the biopsy specimen, albeit with the addition of abundant mature ganglion cells. The latter were often dysmorphic, binucleate, and immunopositive for SYP and/or NFP. Vimentin immunoreactivity was abundant and exceeded GFAP staining within the desmoplastic tissue. Tubular structures were a less prominent feature than in the biopsy specimen. Zones of gangliocytomatous tissue containing only

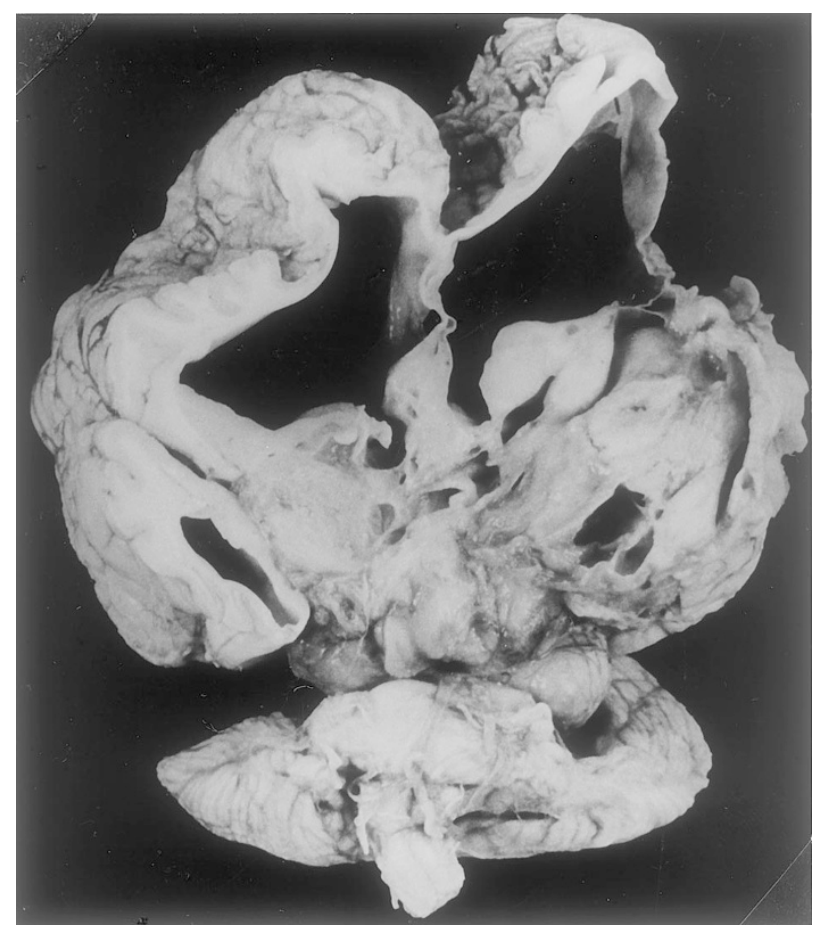

FIGURE 5. Autopsy specimen at age 6 years. The brain is cut in coronal plane through the diencephalon and temporal lobe. Note extensive replacement of bilateral deep nuclear structures by the tumor which bulges to the ventral aspect of the cerebrum. The lesion also compresses the upper brain stem and cerebellar vermis on the left. 
dysplastic neurons within neuropil were seen in the leptomeninges. In addition, undifferentiated cells of small to intermediate size and round to oval configuration were present in subpial zones. Moderate to high in cellularity and featuring cytologic atypia, they exhibited only rare mitoses, a very low Ki-67 LI, and neither GFAP nor SYP immunoreactivity.

Devoid of desmoplasia, intraparenchymal portions of the tumor were composed of astrocytes, neurons, and undifferentiated cells. Overall, the appearance was that of an intimate admixture of ganglioglioma and astrocytoma. Gangliogliomatous regions featured mature, occasionally bi- or multinucleate ganglion cells in a fine fibrillary background (Fig. 6a). Also seen were occasional ballooned cells, axonal spheroids, and perivascular lymphocytic cuffs. The glial element resembled pilocytic astrocytoma (Fig. 6B) and consisted mainly of GFAP-positive astrocytes, including compact piloid cells associated with Rosenthal fibers and microcystic tissue containing granular bodies (Fig. 6C). At the periphery of the tumor, glial-appearing cells, some intermediate in differentiation and showing only occasional GFA positivity, were seen to permeate brain parenchyma.

Approximately two thirds of the left optic nerve was expanded and replaced by tumor that infiltrated both its fiber pathways and interstitial connective tissue septa. Also involved was the leptomeningeal sheath of the nerve, which showed typical features of DIG (Fig. 6D). Within the optic nerves, the infiltrates consisted of neuroepithelial cells of indeterminate type, only occasionally posi-
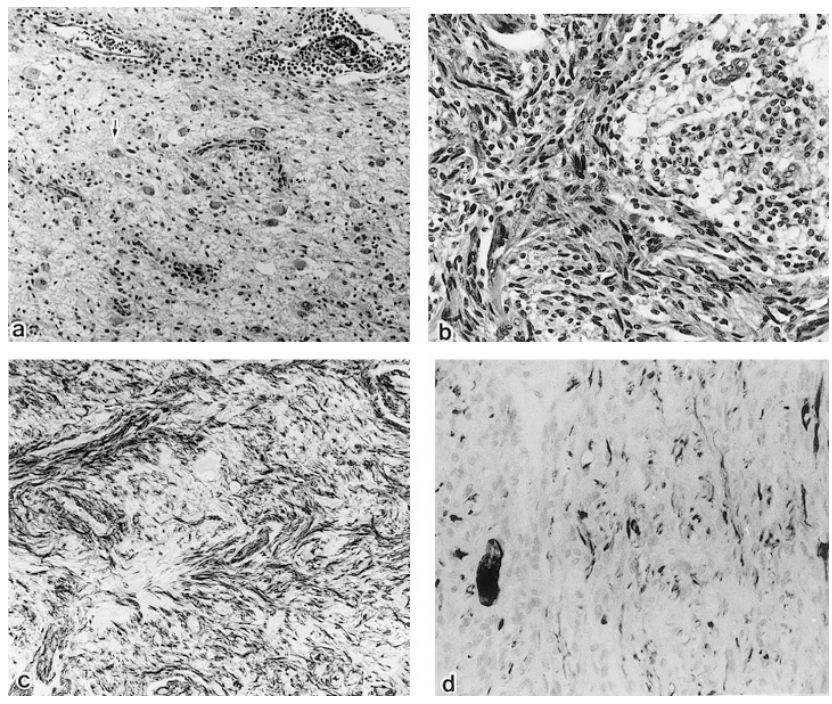

FIGURE 6. A, Gangliogliomatous portion of the lesion. A binucleated neuron (arrow) and perivascular lymphocytes are present. B, A biphasic component resembling ordinary pilocytic astrocytoma. C, GFAP immunoreactivity in the compact, predominantly astrocytic portion of the tumor. D, Neurofilament protein-immunopositive ganglion cell and processes in the leptomeninges of the left optic nerve. tive for GFAP or NFP, and unassociated with desmoplasia.

\section{DISCUSSION}

The histologic findings of the biopsy specimen obtained at age $31 / 2$ months clearly met the definition of DIG $(2,4)$. Neuroepithelial cells and fibroblasts were intimately admixed, and desmoplasia was conspicuous. Astrocytic differentiation was evidenced by GFAP immunoreactivity and presence of dense intermediate filaments. As is often the case, neuronal differentiation could only be confirmed by immunohistochemical and ultrastructural methods (2).

The most distinctive finding in our biopsy was the formation of tubular structures covered by a common basal lamina and containing astrocytes, possible Schwann cells, and primitive-appearing cells. The finding in DIG of what appear to be Schwann cells and/or cells exhibiting pinocytic vesicles has been reported previously $(5,6)$, as has mesoaxon formation (5). The latter is the most distinctive feature for Schwann cells and has a strict morphologic definition (7). Neither the mesaxonlike structures previously reported (5), nor those noted in our case, meet these stringent criteria (7). Thus, the issue of whether Schwannian differentiation occurs in DIG remains unsettled. Although the intimate coexistence of astrocytes and Schwannlike cells within the tubular structures of DIG suggests that they have a common origin, astrocytes are neural-tube derivatives, and Schwann cells are known to originate from neural crest. This seeming inconsistency might be resolved if the source of the neoplastic cells is more primitive, perhaps related to the neural plate. An alternative explanation is that what appear to be astrocytes expressing GFAP within the tubes are actually schwannian in nature. Schwann cells are known to produce this protein (8). It is also relevant that Schwann cells are thought to contribute to conventional ganglioglioma. Supporting evidence include the presence of "mesenchymal cells" surrounded by basal lamina, ones reminiscent of satellite cells in peripheral ganglia (9). The question of whether Schwann cells are a feature of DIG and of conventional ganglioglioma clearly requires further study.

When compared with previously reported cases, the present DIG is somewhat unique in terms of its location, growth pattern, and negative clinical outcome. Serial CT scans demonstrated growth of the deep, centrally situated tumor from the left hypothalamic region into the opposite diencephalon during the first 2 years, followed thereafter by simple fluid accumulation within cysts and progressive ventricular dilatation. Only two postsurgical deaths 
$(3,10)$ and two multiple recurrences of DIG/DIA (2, 11) have been previously reported. Despite its exceptional topography, large size, and degree of leptomeningeal involvement, the histologic grade of our tumor was at most intermediate, the bulk of the lesion being composed of typical low-grade components. Thus, our patient's death of hypothalamic dysfunction and respiratory insufficiency was less due to its innate aggressiveness than to the tumor's deep, central location and to brainstem compression. The biologic potential of immatureappearing, brain-invasive cells at the periphery of the tumor remains uncertain.

Unlike the biopsy specimen, in which only immunostains and electron microscopy confirmed neuronal differentiation, the autopsy specimen clearly showed elements of (1) classic ganglioglioma with readily identifiable ganglion cells showing occasional NFP immunoreactivity and (2) NFP-positive cells in the typical predominantly leptomeningeal DIG. Astrocytic cells also differed in their appearance, being tube-associated in the biopsy specimen and part of a solid, intraparenchymal proliferation of pilocytic astrocytes in the autopsy specimen. These differences in appearance between the biopsy and autopsy specimens can be viewed either as evidence of a maturation phenomenon or as a result of inadequate sampling. Although it is nevertheless possible that over the 6 -year clinical course both the ganglion-like and astrocytic cells matured to ganglion cells and "better differentiated" pilocytic astrocytes, it is also possible that the lesion is essentially biphasic, in other words, a mixed ganglioglioma/DIG. The issue remains unsettled.

In the autopsy specimen, mesenchymal tissues were represented by fibroblasts identical to those seen in reactive processes (12). These were restricted to leptomeningeal portions of the tumor and contributed to its desmoplastic character. Instead, neuroepithelial tissues composed of astrocytic, neuronal and somewhat primitive-appearing cells were present in the both mesenchymal and parenchymal portions, and exhibited a permeative pattern of growth. Our findings would indicate that in DIG, it is fibroblasts rather than neuroepithelial cells that contribute to collagen production. The fact that astrocytes and Schwann cells do not normally produce interstitial (Type I) collagen $(13,14)$ supports this conclusion. Thus we consider the mesenchymal components of DIG to be reactive in nature.

The association of DIG with more conventional ganglion cell tumor has been reported previously. The three cases include an adolescent and a young adult whose DIGs exhibited components of classic ganglioglioma (15), and a 4-year-old boy in whose tumor a gangliocytoma element was also evident
(16). Again, given the relatively advanced age of these patients, the ganglioglioma/cytoma components may be explained by an interval of progressive maturation. In any case, their concurrence underscores the close relation of DIG to conventional ganglion cell tumors.

In conclusion, our study suggests the principal cellular component of DIG is an immature, perhaps neural plate-derived lesion capable of divergent differentiation, not only along neuronal and astrocytic lines, but perhaps toward Schwann cells as well. Its fibroblastic element, presumably derived from leptomeninges, is interpreted as reactive in nature and the source of interstitial collagen. It appears clear that DIG are not only directly related to conventional ganglioglioma, but that the latter may arise in transition from the former. Last, it is not surprising that an occasional DIG, if subtotally resected, is capable of slow progressive growth, and that parenchymal infiltration may prove fatal.

Acknowledgments: The authors thank Dr. Takanori Hirose of Saitama Medical School, Saitama, Japan, and Caterina Giannini of Mayo Clinic, Rochester, Minnesota, for critical review of the manuscript, as well as Ms. R. Umizu and H. Eguti, both of Tokyo Metropolitan Institute for Neuroscience, Fuchu, Japan, for their valuable technical assistance.

\section{REFERENCES}

1. VandenBerg SR, May EE, Rubinstein LJ, Herman MM, Perentes E, Vinores SA, et al. Desmoplastic supratentorial neuroepithelial tumors of infancy with divergent differentiation potential ("desmoplastic infantile gangliogliomas"). Report on 11 cases of a distinctive embryonal tumor with favorable prognosis. J Neurosurg 1987;66:58-71.

2. VandenBerg SR. Desmoplastic infantile ganglioglioma and desmoplastic cerebral astrocytoma of infancy. Brain Pathol 1993;3:275-81.

3. Taratuto AL, Monges J, Lylyk P, Leiguarda R. Superficial cerebral astrocytoma attached to dura. Report of six cases in infants. Cancer 1984;54:2505-12.

4. Taratuto AL, VandenVerg SR, Rorke LB. Desmoplastic infantile astrocytoma and ganglioglioma. In: Kleihues P, Cavenee WK, editors. Pathology and genetics. Tumours of the nervous system. Lyon, France: IARC Press; 2000. p. 99-102.

5. Gambarelli D, Hassoun J, Choux M, Toga M. Complex cerebral tumor with evidence of neuronal, glial and Schwann cell differentiation: a histologic, immunocytochemical and ultrastructural study. Cancer 1982;49:1420-8.

6. Ng TH, Fung CF, Ma LT. The pathological spectrum of desmoplastic infantile gangliogliomas. Histopathology 1990;16: 235-41.

7. Thomas P, Ochoa J, Berthold C, Carlstedt T, Corneliuson O. Microscopic anatomy of the peripheral nervous system. In: Thomas P, Dyck P, editors. Peripheral neuropathy. 3rd ed. Philadelphia: Saunders; 1993. p. 28-92.

8. Memoli VA, Brown EF, Gould VE. Glial fibrillary acidic protein (GFAP) immunoreactivity in peripheral nerve sheath tumors. Ultrastruct Pathol 1984;7:269-75. 
9. Rubinstein LJ, Herman MM. A light- and electronmicroscopic study of a temporal-lobe ganglioglioma. J Neurol Sci 1972;16:27-48.

10. Taratuto AL, Sevlever G, Schultz M, Gutierrez M, Monges J, Sanchez M. Desmoplastic cerebral astrocytoma of infancy (DCAI). Survival data of the original series and report of two additional cases, DNA, kinetic and molecular genetic studies [abstract]. Brain Pathol 1994;4:423.

11. Setty SN, Miller DC, Camras L, Charbel F, Schmidt ML. Desmoplastic infantile astrocytoma with metastases at presentation. Mod Pathol 1997;10:945-51.

12. Reed ML, Jacoby RA. Cutaneous neuroanatomy and neuropathology. Normal nerves, neural-crest derivatives, and benign neural neoplasms in the skin. Am J Dermatopathol 1983;5:335-62.
13. Bunge MB, Williams AK, Wood PM, Uitto J, Jeffrey JJ. Comparison of nerve cell and nerve cell plus Schwann cell cultures, with particular emphasis on basal lamina and collagen formation. J Cell Biol 1980;84:184-202.

14. Paulus W, Roggendorf W, Schuppan D. Immunohistochemical investigation of collagen subtypes in human glioblastomas. Virchows Arch A Pathol Anat Histopathol 1988;413: 325-32.

15. Kuchelmeister K, Bergmann M, von Wild K, Hochreuther D, Busch G, Gullotta F. Desmoplastic ganglioglioma: report of two non-infantile cases. Acta Neuropathol 1993; 85:199-204.

16. Ishida $\mathrm{Y}$, Tanuma M. Desmoplastic gangliglioma. Brain Tumor Pathol 1991;8:79-83. 